\title{
The Kyoto Collection of Human Embryos and Fetuses: History and Recent Advancements in Modern Methods
}

\author{
Yutaka Yamaguchi ${ }^{a}$ Shigehito Yamada ${ }^{a}$ b \\ ${ }^{a}$ Congenital Anomaly Research Center, Kyoto University Graduate School of Medicine, Kyoto, Japan; \\ ${ }^{\mathrm{b}}$ Human Health Sciences, Kyoto University Graduate School of Medicine, Kyoto, Japan
}

\section{Keywords}

Anatomy · Embryo/fetus · Embryology human · Histology human · Image analysis · Image analysis medical · Magnetic resonance imaging

\begin{abstract}
The Kyoto Collection of Human Embryos and Fetuses, the largest collection of human embryos worldwide, was initiated in the 1960s, and the Congenital Anomaly Research Center of Kyoto University was established in 1975 for longterm storage of the collection and for the promotion of research into human embryonic and fetal development. Currently, the Kyoto Collection comprises approximately 45,000 specimens of human embryonic or fetal development and is renowned for the following unique characteristics: (1) the collection is considered to represent the total population of fetal specimens nationwide in Japan, (2) it comprises a large number of specimens with a variety of external malformations, and (3) for most specimens there are clinical and epidemiological data from the mothers and the pregnancies concerned. Therefore, the specimens have been used extensively for morphological studies and could potentially be used for epidemiological analysis. Recently, several new approaches such as DNA extraction from formalin-fixed speci-
\end{abstract}

mens or geometric morphometrics have been adopted and it is to be expected that further technological advances will facilitate new studies on the specimens of the Kyoto Collection as well as of other human embryo collections worldwide. Permanent preservation of the Kyoto Collection is, therefore, of paramount importance so that it will continue to contribute to human embryological studies in the future.

(c) 2018 S. Karger AG, Basel

\section{Initiation of the Kyoto Collection}

Human embryo and fetal specimens have important roles in studies on human embryology. The Kyoto Collection, one of the most well-known collections of human embryos and fetuses, was initiated by Prof. Hideo

\section{Abbreviations used in this paper}

3-D 3-dimensional

CT computed tomography

MRI magnetic resonance imaging

MRM magnetic resonance microscopy

PCX-CT phase contrast X-ray computed tomography

\section{KARGER}

(c) 2018 S. Karger AG, Basel 
Nishimura at the Department of Anatomy, Faculty of Medicine, Kyoto University, circa 1960. There were 2 reasons for initiating this collection. First, reliable information on human embryonic development, both normal and abnormal, was meager because a majority of the data was derived from few specimens obtained from spontaneous abortions or from medical termination of pregnancy among mothers with pathological conditions. Second, numerous recorded induced abortions $(\sim 1.1 \mathrm{mil}-$ lion/year) increased from 1952 because of the revision of the Japanese Eugenic Protection Law that allowed qualified gynecologists to terminate pregnancy for sociomedical reasons [Nishimura et al., 1968]. The law was revised to the Maternal Protection Law of Japan in 1996, and until now fetal specimens have been harvested in accordance with this law. Hence, large-scale collection of human embryos was carried out in cooperation with many obstetricians. Consequently, 34,270 embryos and 3,852 fetuses were collected from 1962 to 1974, and in 1975 the Congenital Anomaly Research Center of Kyoto University was established with Prof. Nishimura as the first Director.

\section{Unique Characteristics of the Kyoto Collection}

The Kyoto Collection is the largest collection of human embryos worldwide, with the following major characteristics. First, it is considered to represent the total population of Japanese fetuses nationwide because the attending obstetricians were not involved in examining the aborted fetuses and the collection of embryos was not biased by their outcome [Nishimura et al., 1974, 1975]. Second, the collection comprises specimens with various external malformations. Using these data, Nishimura et al. [1968] reported that the incidence of malformations in embryos were more frequent than that in infants. In addition, Shiota [1991], the former center director of the Congenital Anomaly Research Center, reported the estimated frequency of malformations and the mortality rate based on an empirical scrutiny of the Kyoto Collection; he reported that embryos with severe malformations were prone to spontaneous abortion at high rates. Third, most specimens were accompanied by clinical and epidemiological information on the corresponding pregnancies and mothers. Kameda et al. [2012] conducted a statistical analysis based on epidemiological data to determine potential causative associations between maternal factors and congenital anomalies. These characteristics render the Kyoto Collection valuable.

The Kyoto Collection of Human Embryos and Fetuses
However, the expansion of collections of human embryos has become very difficult owing to changes in artificial abortion techniques; therefore, human fetuses and embryos are limited valuable specimens. Hence, permanent preservation of this collection is warranted to contribute to further studies in human embryology and genetics and for new imaging techniques. Specimens are imaged and digitalized via various imaging modalities such as magnetic resonance imaging (MRI) or computed tomography (CT). Thus, 3-dimensional (3-D) structures can be analyzed in noninvasive ways and a stereoscopic image database can be constructed.

\section{Available Data of Specimens}

The specimens stored in the Congenital Anomaly Research Center of Kyoto University have been used for morphological or epidemiological studies. Currently available specimens are primarily sets of serial sections and formalin-fixed specimens stored in bottles (Fig. 1). In particular, formalin-fixed specimens have been imaged using various modalities. Here, we briefly introduce those that are currently used.

\section{Serial Sections}

In total, 1,044 sets of serial sections (547 cases of normal embryos and 497 cases of embryos with an abnormal external appearance) are now stored in the microscopy room of this center (Fig. 1). These sections (Fig. 2a) are used for detailed histological analysis and 3-D reconstruction. For example, Saitsu et al. [2007] reported 3 cases of human embryos with myeloschisis, wherein the open primary neural tube and the closed secondary neural tube overlap dorsoventrally, and that the axially condensed mesenchyme played some roles in normal and abnormal development of the human posterior neural tube. Those sections were generated approximately 30 40 years ago. Therefore, some stains have faded, and some glass slides have degraded with time. Hence, some digitization projects have been initiated to acquire images of specimens, using several imaging modalities, i.e., not only high-resolution scanners but also the commercial flatbed scanner. Though the commercial flatbed scanner has a lower resolution of $4,800 \mathrm{dpi}$, it scans serial sections comparatively quickly and generates relatively smaller and suitable data. Miyazaki et al. [2018] also carried out a similar study in the Blechschmidt Collection of University of Göttingen (Göttingen, Germany), using a commercial flatbed scanner.

Cells Tissues Organs 2018;205:314-319 315 
Fig. 1. a Microscopy room. Numerous sets of serial sections $(\sim 1,000$ embryos $)$ are stored. b Storage lockers of the specimen room. c Formalin-fixed embryo specimens. Sample No. 33317.
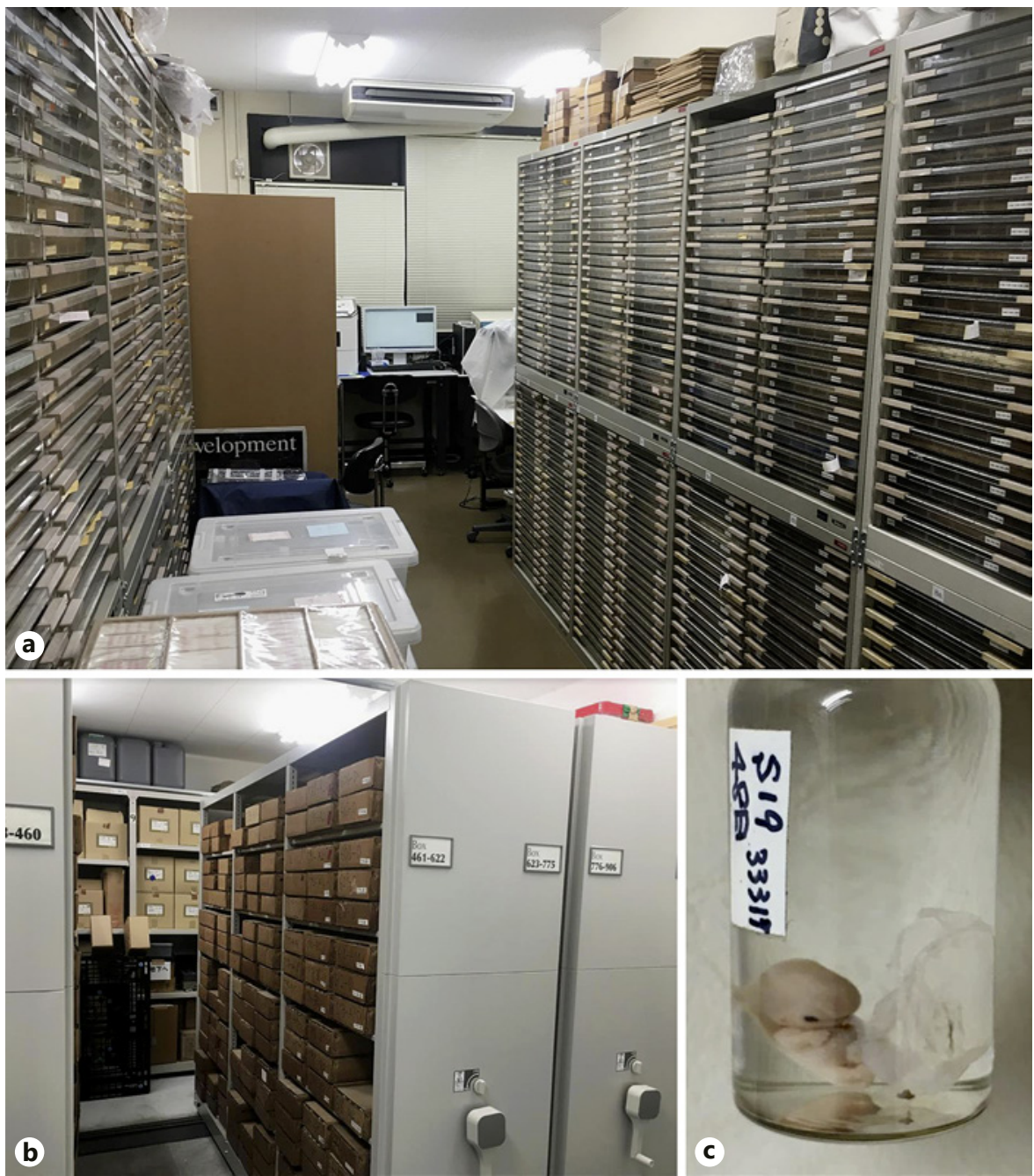

\section{MR Microscopy}

Serial sections are suitable for detailed observation of internal structures of embryos. However, a high level of skill is required to obtain complete sets of serial sections, and generation of such sections is associated with loss of whole embryos owing to sectioning. The Kyoto Human Embryo MR Microscopy Project was initiated to observe internal structures in a noninvasive manner in May of 2003. Consequently, 3-D MR microscopic images of 1,204 embryos were acquired at the spatial resolution of $40-150 \mu \mathrm{m}^{3}$ using a 2.34 -T MRM (super-parallel MRM) at the Institute of Applied Physics, University of Tsukuba [Matsuda et al., 2007] (Fig. 2b). This number is unparalleled in other studies; therefore, these data were used for different organogenesis studies. Moreover, a morphological database was generated on the basis of MRM images from 2005 to 2010 [Yamada et al., 2006].

\section{Phase Contrast X-Ray CT}

Noninvasive 3-D imaging of embryos was possible using a 2.34-T MRM. However, the spatial resolution of MRM is approximately $40 \mu \mathrm{m}^{3}$ and it is not suitable for analysis of small embryos. Therefore, imaging studies using phase contrast X-ray CT (PCX-CT) are underway. PCX-CT is a relatively newer imaging modality in which the X-rays are used as electric waves, which provide information regarding amplitude and phase. When an Xray penetrates a sample, its amplitude is decreased and the phase is shifted. Thus, spatial resolutions of up to $9 \mu \mathrm{m}^{3}$ were achieved and finer 3-D images were obtained (Fig. 2c) [Yoneyama et al., 2006; Kanahashi et al., 2016].

\section{Magnetic Resonance Imaging}

MRM and PCX-CT can only image embryonic specimens because of their imaging range. The field of view of 

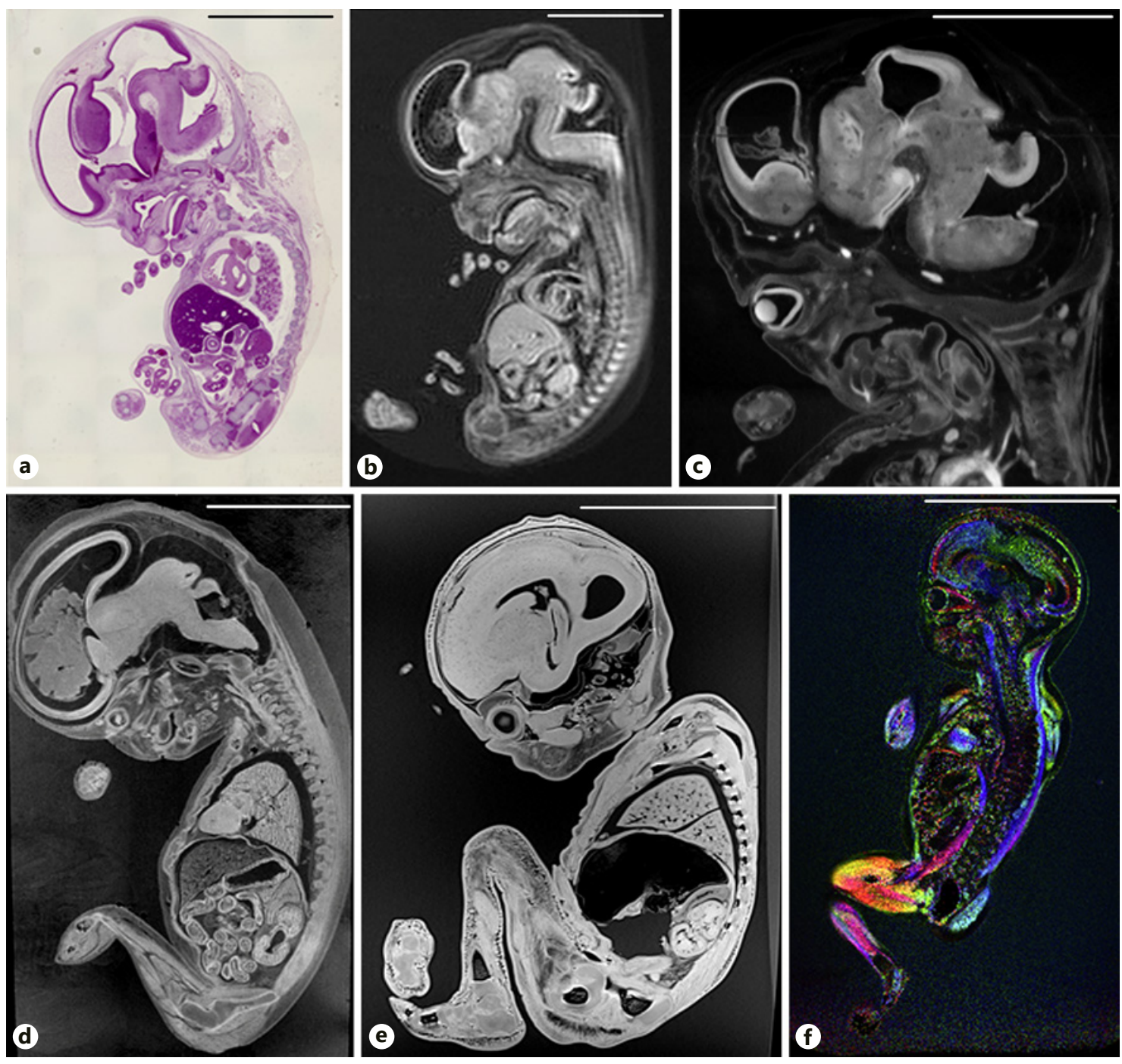

Fig. 2. a Serial histological section of a human embryonic specimen stained with hematoxylin and eosin. b MR microscopy image of a human embryo. c Phase contrast X-ray CT image of a human embryo. d T1-weighted MR image of a human fetus, using a 7-T MR scanner. e T2-weighted MR image of a human fetus, using a 3-T MR scanner. f Color map of a human fetus, using a 3-T MR scanner. Sample No. 9026 (a), 52817 (b), 52817 (c), 52201 (d), $91473(\mathbf{e})$, and $53604(\mathbf{f})$. Scale bars, $5 \mathrm{~mm} \mathrm{(a-c),} 1 \mathrm{~cm}(\mathbf{d})$, and $5 \mathrm{~cm}(\mathbf{e}, \mathbf{f})$.

MRM is $38.4 \times 19.2 \mathrm{~mm}$ and that of PCX-CT is $60 \times 30$ $\mathrm{mm}$ at $17 \mathrm{keV}$. Although they are suitable for imaging of embryos, they cannot image large specimens such as fetuses. The Kyoto Collection has not only embryos but also many fetal specimens. Therefore, to acquire images of these fetal specimens, some imaging studies are currently underway using MRI. Currently, 2 MRI apparatus are used, i.e., a 7-T preclinical MRI (BioSpec 70/20 USR; Bruker BioSpin MRI GmbH, Ettlingen, Germany) at the Biomedical Engineering Laboratory, Kyoto University, and a clinical 3-T MRI (Siemens Healthcare, Erlangen,
Germany) at the Kyoto University Hospital. A 7-T MRI has a spatial resolution of $35.4-54.7 \mu \mathrm{m}^{3}$ and is suitable for the early stage of the fetal period, e.g., approximately 20-60 mm in crown-rump length (Fig. 2d). However, 3-T MRI has a spatial resolution of $200 \mu \mathrm{m}^{3}$ and a lower resolution than 7-T MRI. Larger fetal specimens can be imaged using 3-T MRI (Fig. 2e). To date, there are imaging data for fetal specimens of up to $236 \mathrm{~mm}$ in crown-rump length. Using these data, Yamaguchi et al. [2018] reconstructed 3-D atlas models of the developing fetal brain. In addition, diffusion-weighted imaging can be performed 


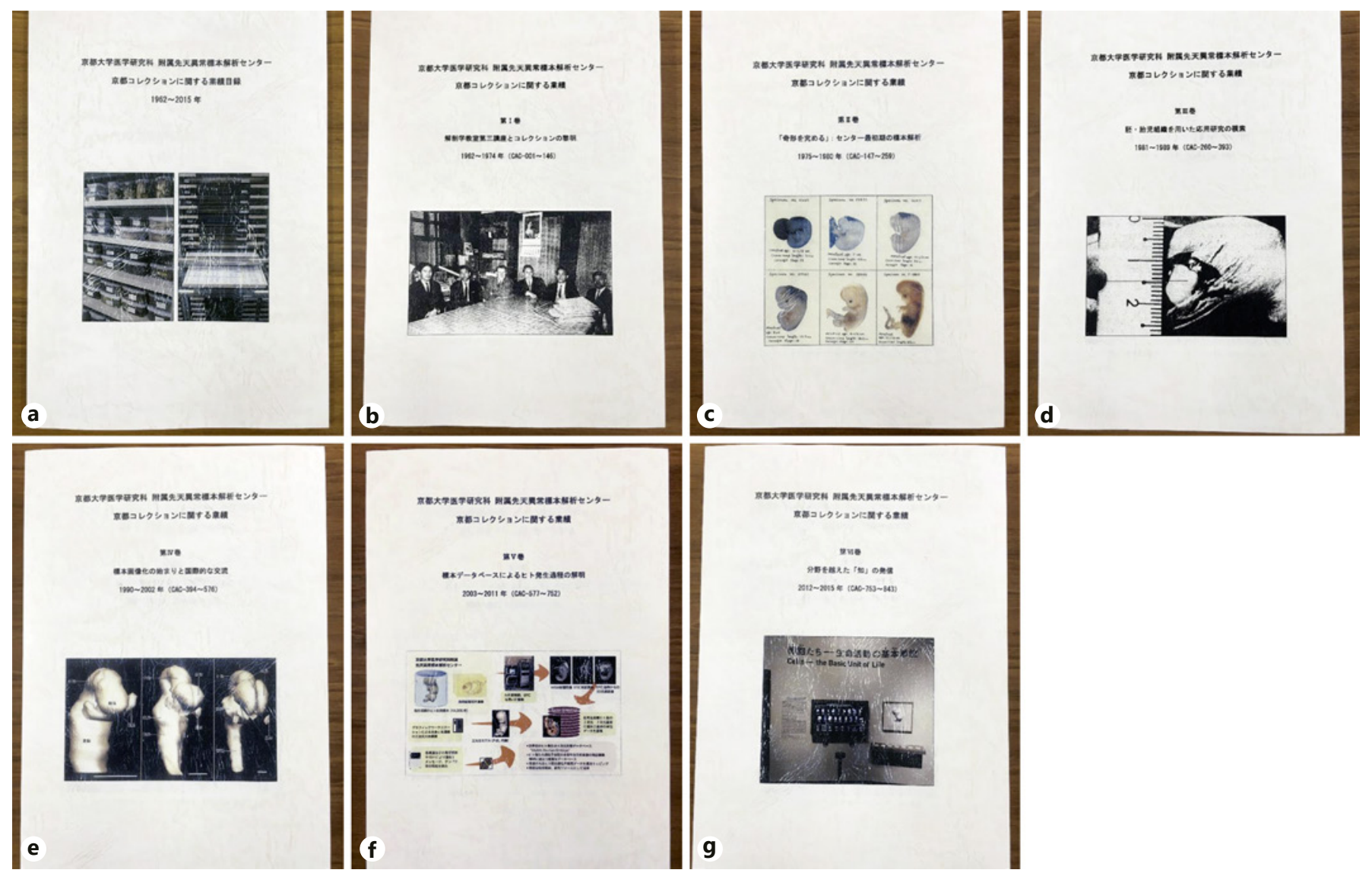

Fig. 3. One catalog and 6 books of research compilations from 1962 to 2015 . a A catalog contains 843 achievements from 1962 to 2015, such as abstracts of many societies or conferences, reviews or research articles, books, and news stories. b-g Research compilations from 1962-1974, 1975-1980, 1981-1989, 1990-2002, 2003-2011, and 2012-2015, respectively.

using 3-T MRI; therefore, muscle and brain fibers can be extracted using diffusion-weighted imaging (Fig. 2f). Currently, imaging at a spatial resolution of $100 \mu \mathrm{m}^{3}$ using 3-T MRI is underway.

\section{Studies Using Specimens from the Kyoto Collection}

The Kyoto Collection has numerous resources, not only serial sections but also imaging data. Using these resources, several studies have been conducted thus far and all studies from 1962 to 2015 have been compiled in 6 books of the research compilations at the 40th anniversary symposium in 2015 (Fig. 3). These pages are part of a reprint which is used in our center. Currently, preparations for uploading this catalog online are underway. Information regarding our center is provided in these catalogs.
Moreover, at present, new studies using specimens from the Kyoto Collection have increased. Regarding serial sections, the information has been entered into the Digital Embryology Consortium. This consortium was established in 2014, and the objective of this international partnership was to digitize, preserve, and make available for researchers the major embryology histological collections [Digital Embryo Consortium, 2015]. In addition, serial sections have certain limitations such as shrinkage and distortion of the sections [Yamada et al., 2007]; therefore, Kajihara et al. [2017] attempted to solve these problems using the technique of feature-based nonrigid registration. Regarding imaging data, some morphological studies are underway. For example, Katsube et al. [2017] reported that the maturation periods of the shapes of the anterior nasal spine and the nasal septum were approximately 12 and 14 weeks of gestation, using 
geometric morphometrics. However, Nagai et al. [2016] attempted to extract genomic DNA from human embryos after long-term preservation in formalin and Bouin's fixative. Stable DNA extraction from human embryonic specimens of this collection can facilitate various studies to elucidate pathomechanisms underlying congenital anomalies.

Thus, technological advancements have facilitated new studies using human embryo collections. Therefore, permanent preservation of the Kyoto collection is warranted. It is hence desirable that this collection will continue to contribute to human embryological studies in the future.

\section{Acknowledgement}

We thank the collaborating obstetricians for their cooperation in supplying fresh specimens. We gratefully acknowledge Chigako Uwabe and Hiromi Yasuda for contributing to the Kyoto Collection of Human Embryos. We thank Editage (www.editage.jp) for providing English language support. Out studies have been approved by the Ethics Committee of the Graduate School of Medicine and the Faculty of Medicine of Kyoto University (approval No. E986, G167, G377, G736, R0316, R0347, and R0989-1).

\section{Disclosure Statement}

There are no conflicts of interests to declare.

\section{References}

Digital Embryology Consortium (2015) Digital Embryology Consortium. https://humanembryology.org (accessed April 9, 2018).

Kajihara, T., T. Funatomi, H. Kubo, H. Makishima, S. Yamada, T. Aoto, Y. Mukaigawa (2017) Feature-based non-rigid registration of serial section images by blending rigid transformations. The 4th Asian Conf Pattern Recognit, Nanjing.

Kameda, T., S. Yamada, C. Uwabe, N. Suganuma (2012) Digitization of clinical and epidemiological data from the Kyoto Collection of $\mathrm{Hu}-$ man Embryos: maternal risk factors and embryonic malformations. Congenit Anom 52: 48-54.

Kanahashi, T., S. Yamada, M. Tanaka, A. Hirose, C. Uwabe, K. Kose, A. Yoneyama, T. Takeda, T. Takakuwa (2016) A novel strategy to reveal the latent abnormalities in human embryonic stages from a large embryo collection. Anat Rec 299: 8-24.

Katsube, M., S. Yamada, R. Miyazaki, Y. Yamaguchi, H. Makishima, T. Takakuwa, A. Yamamoto, Y. Fujii, N. Morimoto, T. Ito, H. Imai, S. Suzuki (2017) Quantitation of nasal development in the early prenatal period using geometric morphometrics and MRI: a new insight into the critical period of Binder phenotype. Prenat Diagn 37: 907-915.

Matsuda, Y., S. Ono, Y. Otake, S. Handa, K. Kose, T. Haishi, S. Yamada, C. Uwabe, K. Shiota (2007) Imaging of a large collection of human embryo using a super-parallel MR microscope. Magn Reson Med Sci 6: 139-146.
Miyazaki, R., H. Makishima, J. Männer, H.G. Sydow, C. Uwabe, T. Takakuwa, C. Viebahn, S. Yamada (2018) Blechschmidt Collection: revisiting specimens from a historical collection of serially sectioned human embryos and fetuses using modern imaging techniques. Congenit Anom DOI: 10.1111/cga.12261.

Nagai, M., K. Minegishi, M. Komada, M. Tsuchiya, T. Kameda, S. Yamada (2016) Extraction of DNA from human embryos after longterm preservation in formalin and Bouin's solutions. Congenit Anom 56: 112-118.

Nishimura, H. (1974) Detection of early developmental anomalies in human abortuses; in Gianantonio CA, Berri GG (eds): Pediatria XIV. Buenos Aires, Editorial Medica Panamericana.

Nishimura, H. (1975) Prenatal versus postnatal malformations based on the Japanese experience on induced abortions in the human being; in Blandeu R (ed): Aging Gamates. Basel, Karger.

Nishimura, H., K. Takano, T. Tanimura, M. Yasuda (1968) Normal and abnormal development of human embryos: first report of the analysis of 1,213 intact embryos. Teratology 1: 281-290.

Saitsu, H., S. Yamada, C. Uwabe, M. Ishibashi, K. Shiota (2007) Aberrant differentiation of the axially condensed tail bud mesenchyme in human embryos with lumbosacral myeloschisis. Anat Rec 290: 251-258.
Shiota, K. (1991) Development and intrauterine fate of normal and abnormal human conceptuses. Congenit Anom 31: 67-80.

Yamada, S., H. Itoh, C. Uwabe, S. Fujihara, C. Nishibori, M. Wada, S. Fujii, K. Shiota (2007) Computerized three-dimensional analysis of the heart and great vessels in normal and holoprosencephalic human embryos. Anat Rec (Hoboken) 290: 259-267.

Yamada, S., T. Takakuwa (2012) Introduction: developmental overview of the human embryo; in Yamada S (ed): Human Embryo. Rijecka, In Tech.

Yamada, S., C. Uwabe, T. Nakatsu-Komatsu, Y. Minekura, M. Iwakura, T. Motoki, K. Nishimiya, M. Iiyama, K. Kakusho, M. Minoh, S. Mizuta, T. Matsuda, Y. Matusda, T Haishi, K. Kose, S. Fujii, K. Shiota (2006) Graphic and movie illustrations of human prenatal development and their application to embryological education based on the human embryo specimens in the Kyoto Collection. Dev Dyn 235: 468-477.

Yamaguchi, Y., R. Miyazaki, M. Kamatani, C. Uwabe, H. Makishima, M. Nagai, M. Katsube, A. Yamamoto, H. Imai, K. Kose, K. Togashi, S. Yamada (2018) Three-dimensional models of the segmented human fetal brain generated by magnetic resonance imaging. Congenit Anom 58: 48-55.

Yoneyama, A., N. Amino, M. Mori, M. Kudoh, T. Takeda, K. Hyodo, Y. Hirai (2006) Non-invasive and time-resolved observation of tumors implanted in living mice by using phase-contrast X-ray computed tomography. Jpn J Appl Phys 45: 1864. 